\title{
Construindo e Averiguando Anéis de Carga
}




\section{Sumário}

Neste projeto pretendemos projetar, construir, testar e averiguar um anel de carga para a realização de testes de fraturas ambientalmente assistidas (FAA) em metais.

As FAA são perigosas, pois elas podem ocorrer sem sinais de corrosão geral e sobre tensões estáticas abaixo do limite de escoamento do material. Assim como nas fraturas causadas por fadigas, estas podem levar a falhas catastróficas sem alerta.

Com isso justificamos motivação para construir um equipamento que de forma econômica possa realizar tais testes e assim os anéis de carga aparecem como uma alternativa. 


\section{Índice}

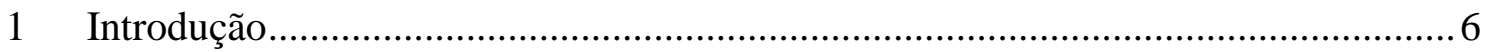

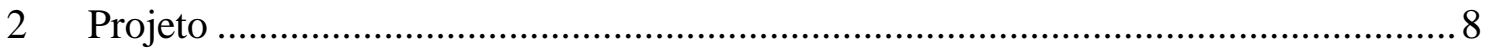

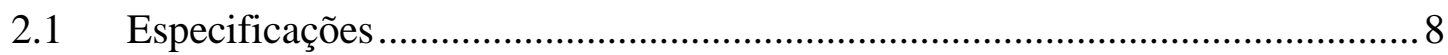

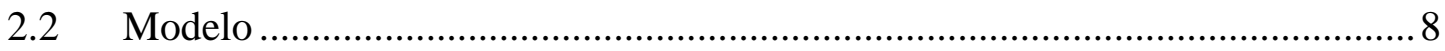

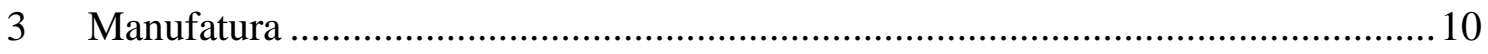

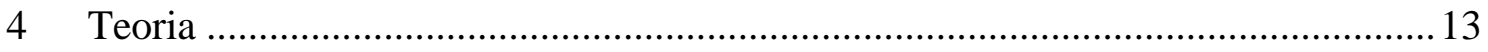

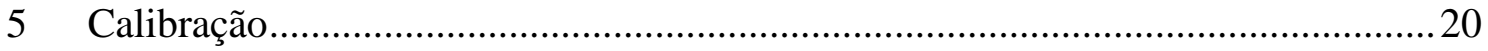

$5.1 \quad$ Sensoriamento

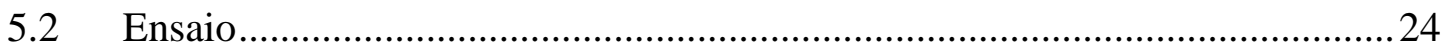

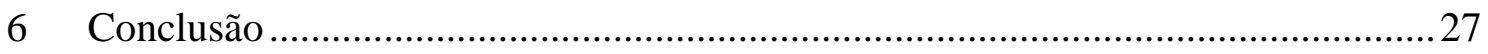

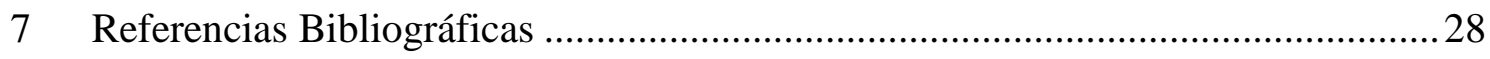




\section{Índice de Figuras}

Figura (1): Exemplo de um Anel de Carga com um relogio comparador........................ 7

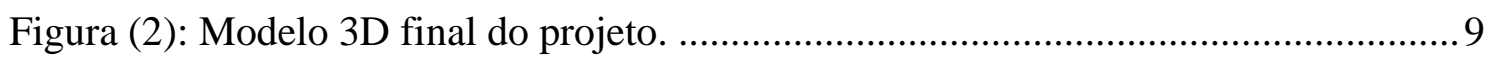

Figura (3): Exemplo de escopo para as garras do projeto........................................ 9

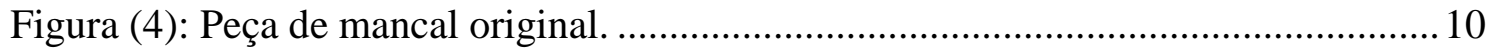

Figura (5): Base da garra apos rosqueamento do pino............................................ 11

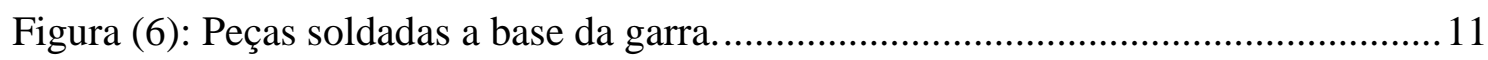

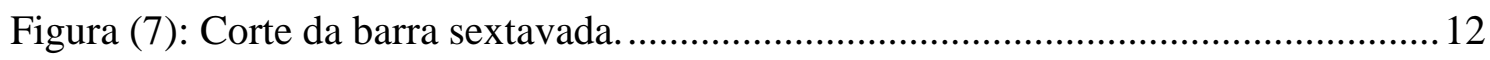

Figura (8): Diagrama de corpo livre do anel de carga. ............................................ 13

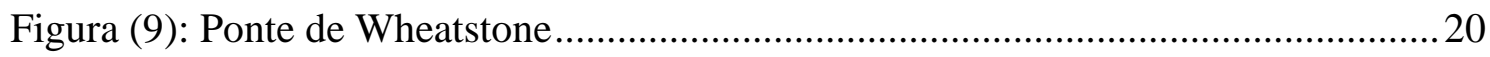

Figura (10): Strain gages e terminal colados no lugar. ........................................... 21

Figura (11): Strain gage conectado ao cabo coaxial. ............................................... 21

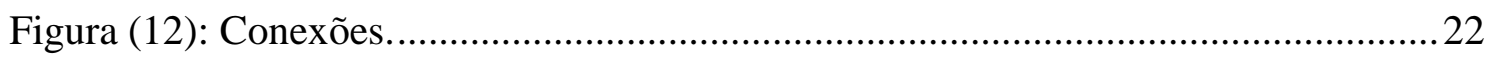

Figura (13): Gráfico da força aplicada com medida. ................................................. 25

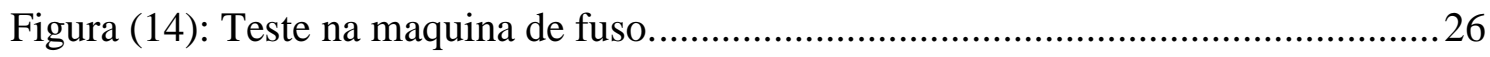


Índice de Tabelas

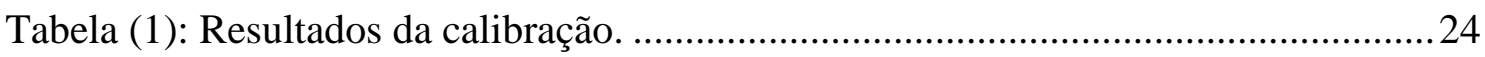

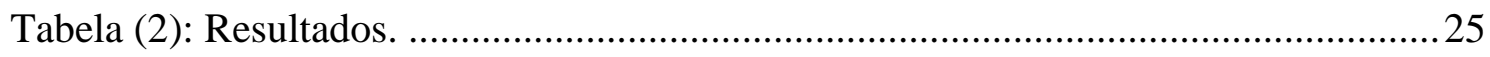




\section{Introdução}

Os anéis de carga (AC) são equipamentos de laboratório utilizados para realizar testes de fratura em ambiente corrosivo em corpos de prova $(\mathrm{CP})$ padrão. Tais testes simulam o que acontece quando o meio ataca o material sobre tensão constante, assim como o que acontecesse com tubulações de óleo que recebem certas quantidades de sulfeto de hidrogênio $\left(\mathrm{H}_{2} \mathrm{~S}\right)$ que causam fragilização no metal por causa do hidrogênio.

Os ACs são constituídos por partes simples e funcionam de modo puramente mecânico e estático, por conta disso eles não possuem partes moveis. A construção de um anel envolve um tubo usinado e cortado em pequenas fatias ao longo de seu comprimento, de acordo com as especificações necessárias para formar o anel em si. Este anel então possui um furo passante ao longo de seu diâmetro, por estes furos passam duas barras roscadas que se conectam a garras que seguram o CP dentro de um compartimento que possui o meio corrosivo. Nestas barras roscadas, na parte exterior do anel, são colocadas porcas, estas são tensionadas de forma que as garras exercem tração no corpo de prova. Assim a parte do anel serve de estrutura para segurar que segura à força exercida no CP.

Esta força causa uma pequena deformação na estrutura do anel e esta deformação pode ser usada para de forma a quantificar a força sofrida pelo corpo de prova. As duas técnicas mais comuns são colar strain gages nos pontos de maior deformação ou usar um relógio comparador, assim como na Figura (1, para medir a diminuição do diâmetro externo do anel.

Com estas duas técnicas existem soluções analíticas que convertem os sinais retirados, o deslocamento do diâmetro ou a deformação do anel, em força exercida sobre o corpo de prova. Com isso é possível quantificar o experimento de forma a seguir um método com rigor cientifico. 


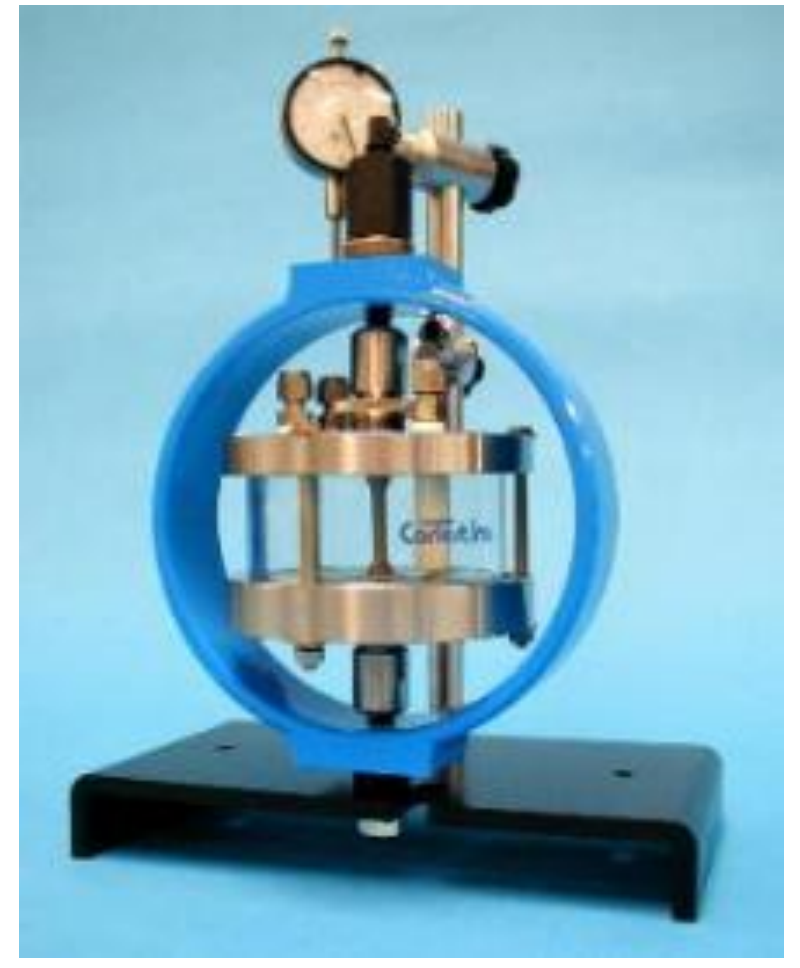

Figura (1): Exemplo de um Anel de Carga com um relogio comparador. 


\section{Projeto}

\subsection{Especificações}

Para o projeto do AC foi sugerido um anel com diâmetro interno de $250 \mathrm{~mm}$, com garras que pudessem segurar um corpo de prova do tipo $\mathrm{C}(\mathrm{T})$ que possua um $w$ de $60 \mathrm{~mm}$ e com sensoriamento eletrônico de strain gages. Para simplificar o projeto, o equipamento foi projetado para ser operado na horizontal, com o CP imerso em um Becker que contenha o agente corrosivo, eliminando assim a necessidade de projetar um compartimento que isole o par ambiente-material do resto do equipamento para evitar danos.

Pensando em usos futuros as garras do equipamento foram desenhadas para serem peças removíveis, de forma que o mesmo AC possa ser utilizado para outros tamanhos ou tipos de CPs.

Outro ponto de projeto importante foi o uso de strain gages ao invés de um relógio comparador. Apesar dos equipamentos eletrônicos serem mais custosos e de manuseio mais complexo, estes foram escolhidos, pois para o uso de um relógio comparador é necessário que existam duas bases paralelas nas extremidades do anel, com os strain gage é possível fabricar um anel que tenha o diâmetro externo circular. Isto é uma enorme vantagem, pois assim o anel pode ser fabricado a partir de tubos usinados em um torno convencional.

\subsection{Modelo}

Para auxiliar na manufatura do anel, primeiro foi criado um modelo de todas as peças em 3D no SolidWorks. A partir disto foram cridos escopos cotados para que fosse possível decidir qual seria a melhor técnica de manufatura das peças.

Este modelo foi alterado algumas vezes durante o processo de fabricação devido à disponibilidade de algumas peças serem maiores que outras. Como por exemplo: porcas e roscas 
que inicialmente seguiam o sistema métrico foram substituídas por equivalentes próximos no sistema americano.

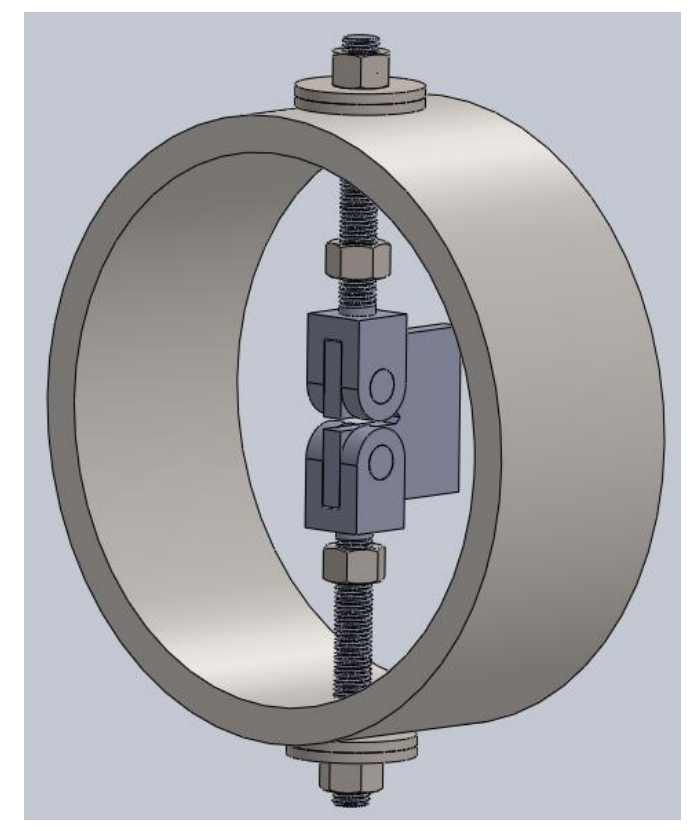

Figura (2): Modelo 3D final do projeto.

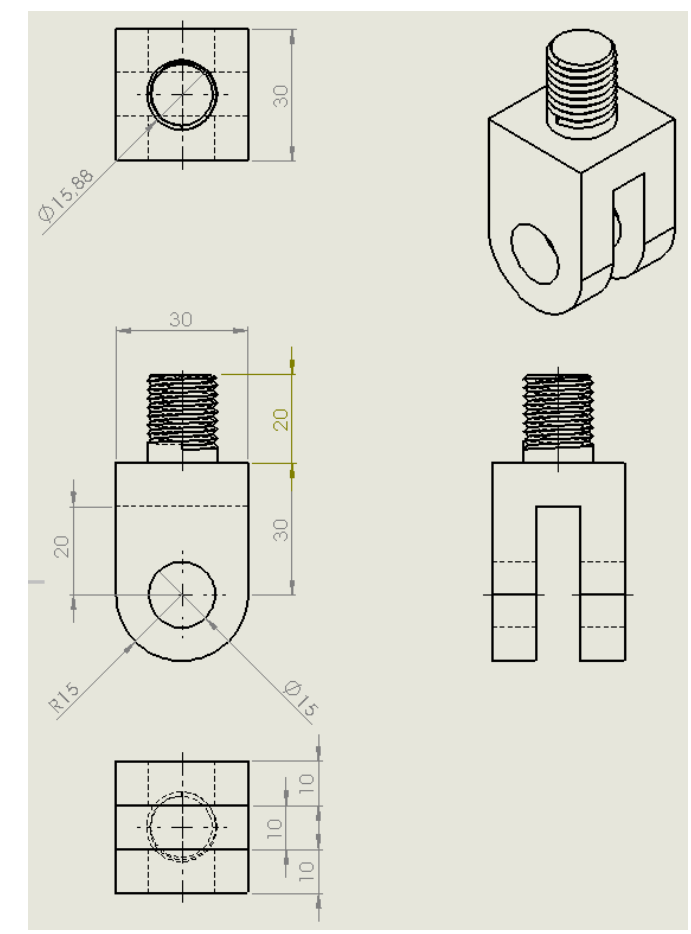

Figura (3): Exemplo de escopo para as garras do projeto. 


\section{Manufatura}

$\mathrm{O}$ anel propriamente dito foi usinado em um torno a partir de uma peça de mancal sucateada de aço 4140, que possui modulo de elasticidade $(E)$ de aproximadamente $200 \mathrm{GPa}$. O diâmetro interno foi aumento até os $250 \mathrm{~mm}$ sugeridos e o externo foi reduzido para que a peça possuísse uma espessura de $15 \mathrm{~mm}$. Em seguida o anel foi perfurado em extremidades opostas no sentido longitudinal com furos de $15,88 \mathrm{~mm}$ de diâmetro, aproximadamente 5/8', para serem compatíveis com os parafusos de mesmo tamanho.

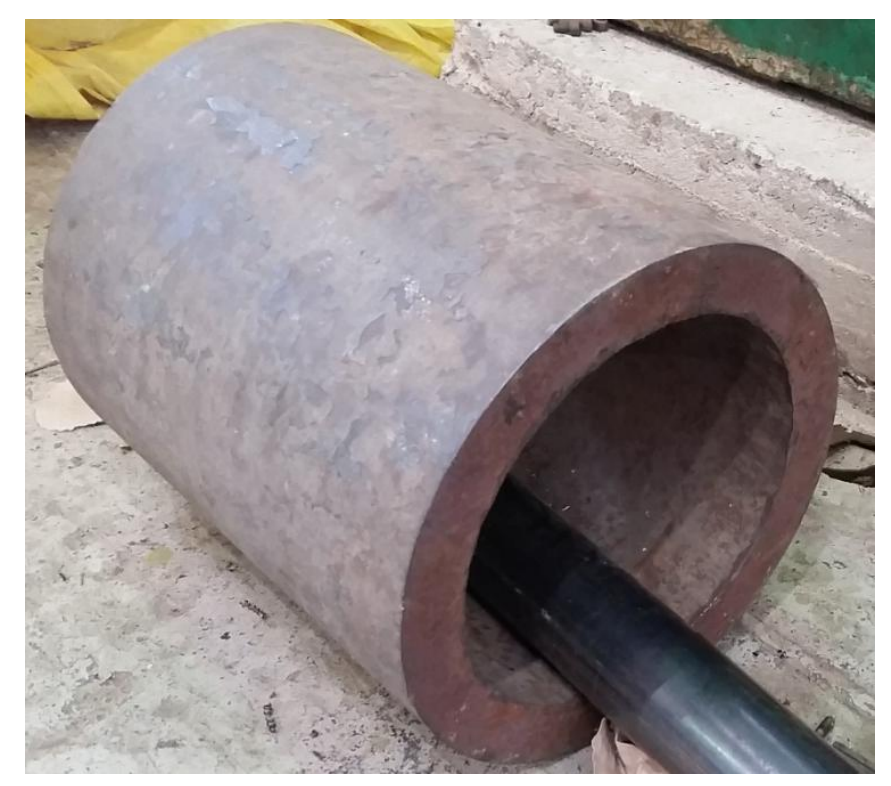

Figura (4): Peça de mancal original.

As garras foram as peças mais complexas do projeto, pois em sua construção foi necessário a soldagem de diversos componentes fabricados separadamente. Primeiro, a partir de um bloco solido de aço 1020 foi usinado uma base quadrada com um pino roscado em sua superfície, assim como mostra a figura a Figura (3) e na Figura (5), depois duas pequenas peças, Figura (6), de aço 1020 foram soldadas na base quadrada para criar a lateral da garra. Em seguida esta foi perfurada com um furo de $15 \mathrm{~mm}$ de diâmetro para acomodar o pino de contenção, isto foi feito posterior a soldagem para garantir o alinhamento do furo. Para finalizar, 
a peça foi lixada para adquirir uma forma abaulada na ponta e também para garantir as dimensões do projeto.

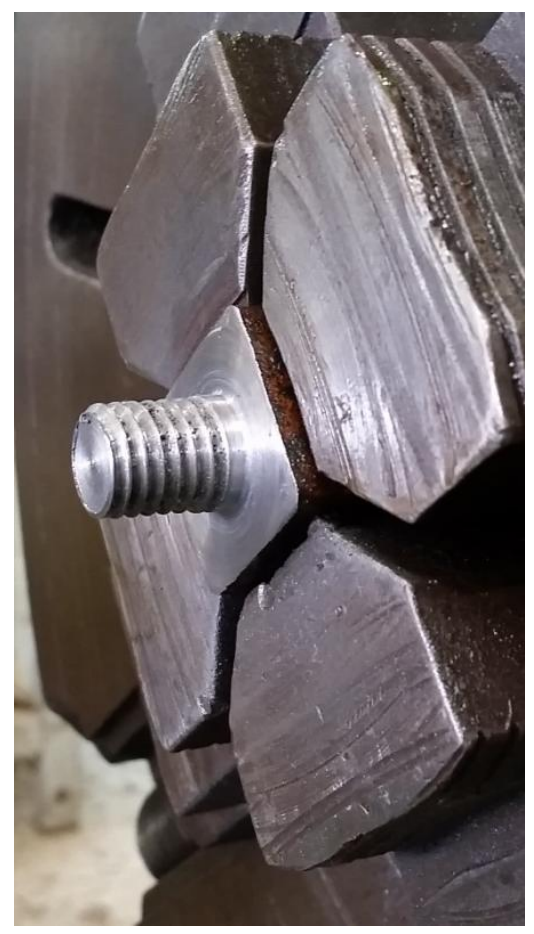

Figura (5): Base da garra apos rosqueamento do pino.

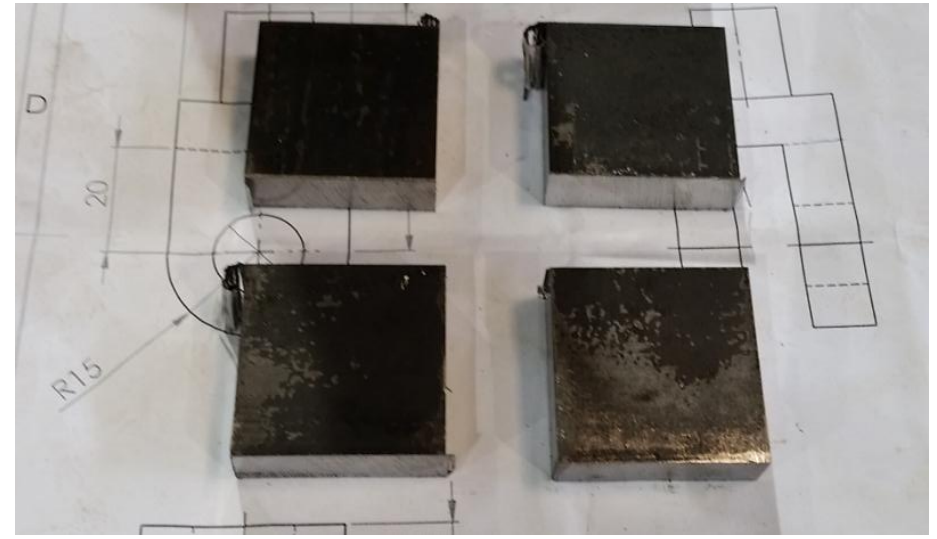

Figura (6): Peças soldadas a base da garra. 
Os pinos passantes foram relativamente fáceis de fazer, pois estes são parafusos de $5 / 8^{\prime}$, com $100 \mathrm{~mm}$ de comprimento com as cabeças cortadas.

As porcas intermediárias, entre as garras e os pinos, foram construídas a partir de uma barra sextavada de aço 1020, cortada, perfurada e roscada internamente. Isto foi feito para que estas possuíssem um comprimento longo que acomodasse a junção do pino com a garra.

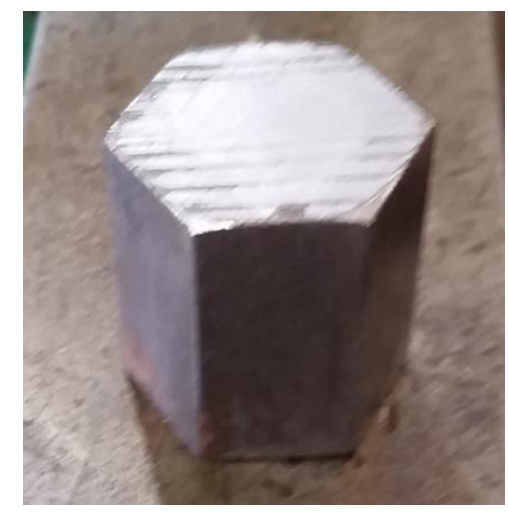

Figura (7): Corte da barra sextavada.

Os pinos de travamento, que passam nos furos das garras foram usinados de aço 4140 também. O diâmetro deles é o mesmo dos furos, $15 \mathrm{~mm}$, entretanto eles possuem uma pequena folga de $0,3 \mathrm{~mm}$, para facilitar o encaixe de CP no equipamento.

As outras porcas e arruelas são todos padrões de 5/8'. 


\section{Teoria}

Assim como dito anteriormente, pretendemos usar strain gages para medir a carga sofrida pelo no corpo de prova. Para tal precisamos encontrar uma relação entre a deformação do anel $(\epsilon)$ com a carga aplicada $(P)$. Entretanto sabemos que a deformação do anel também varia de acordo com a sua posição, usando coordenadas polares podemos definir isto com um ângulo entre a normal e a posição desejada $(\theta)$ e a distancia entre a linha de centro do anel $(y)$. Ou seja, pretendemos encontrar a função $\epsilon(P, \theta, y)$.

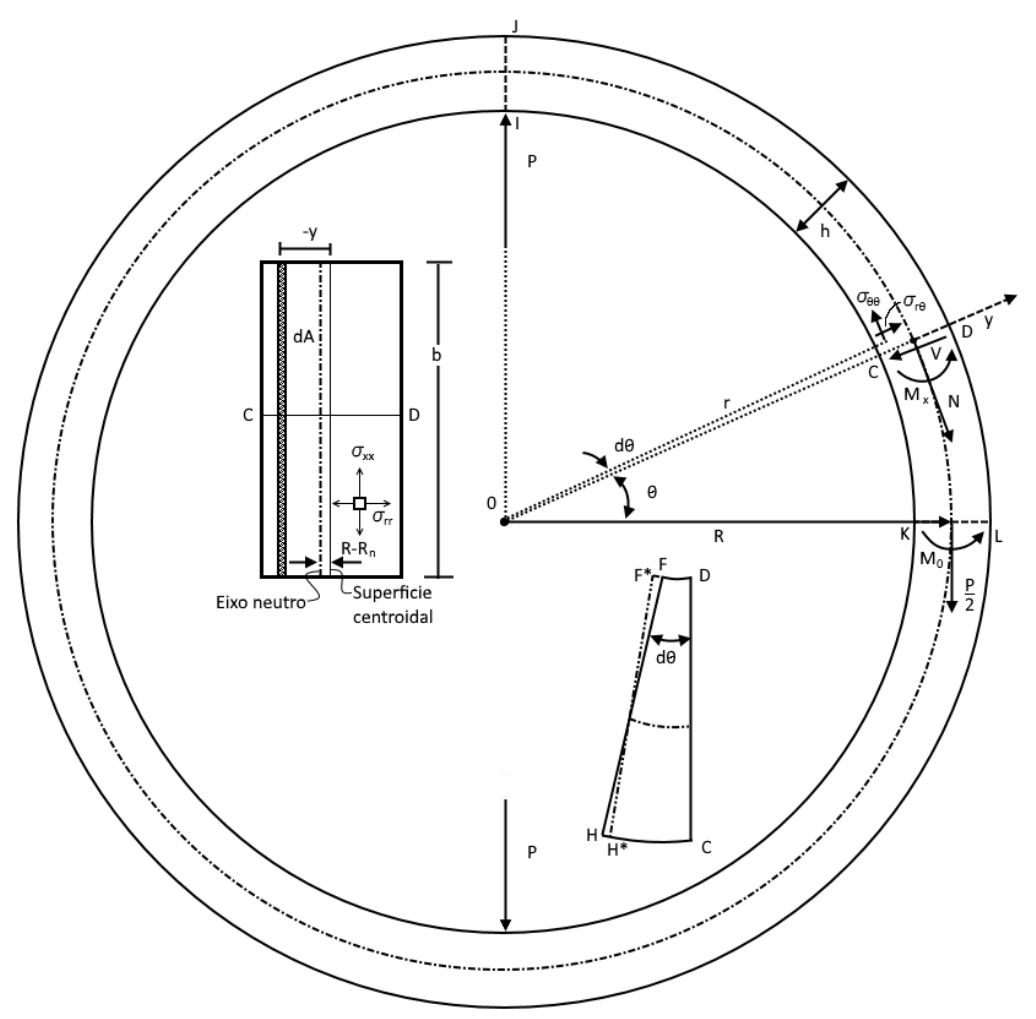

Figura (8): Diagrama de corpo livre do anel de carga.

Com o objetivo de encontrar uma solução para o nosso problema faremos uma analise de um elemento de viga curva, a uma distancia $r$ do centro de curvatura, que está sobre deformação devido a diversas forças e momentos. Para tal, primeiro encontraremos uma relação 
aproximada da distribuição da tensão circunferencial $\left(\sigma_{\theta \theta}\right)$. Um fato importante de se notar é que no calculo de $\sigma_{\theta \theta}$ nos negligenciaremos os efeitos da tensão de cisalhamento $\left(\sigma_{r \theta}\right)$ porque está é muito pequena. Sendo assim começamos com o somatório de forças e momentos neste elemento.

$$
\begin{aligned}
& \sum F_{z}=\int \sigma_{\theta \theta} d A-N=0 \\
& \sum M_{x}=\int \sigma_{\theta \theta}(R-r) d A-M_{x}=0
\end{aligned}
$$

Ou seja:

$$
\begin{aligned}
& N=\int \sigma_{\theta \theta} d A \\
& M_{x}=\int \sigma_{\theta \theta}(R-r) d A
\end{aligned}
$$

Retirando uma fatia da viga curva de tamanho $d \theta$ podemos estudar mais profundamente a deformação sofrida pelo material. Quando esta fatia sofre uma deformação existe uma variação do ângulo de $d \theta,(\Delta(d \theta))$, entretanto essa variação não é linear ao longo da distancia FH e no ponto em que ela é zero encontramos o eixo neutro, neste eixo sabemos que $\sigma_{\theta \theta}=0$. $R_{n}$ é a distancia entre o eixo neutro e o centro de curvatura.

$\mathrm{O}$ alongamento do elemento $\left(d e_{\theta \theta}\right)$ na direção $\theta$ é igual a distancia das faces $\mathrm{FH}$ e $\mathrm{F}^{*} \mathrm{H}^{*}$, e varia linearmente com a distância de $\left(R_{n}-r\right)$. Entretanto a deformação $\epsilon_{\theta \theta}$ é uma função não linear de $r$, já que o elemento de comprimento $r d \theta$ também varia com $r$. Da Figura (8) nos tiramos a seguinte deformação:

$$
\epsilon_{\theta \theta}=\frac{d e_{\theta \theta}}{r d \theta}=\frac{\left(R_{n}-r\right) \Delta(d \theta)}{r d \theta}=\left(\frac{R_{n}}{r}-1\right) \omega
$$

Onde: 


$$
\omega=\frac{\Delta(d \theta)}{d \theta}
$$

Os efeitos de $\sigma_{x x}$ e $\sigma_{r r}$ são pequenos o suficiente para serem considerados desprezíveis e assim podemos levar o problema como se fosse de tensão plana. Sendo assim podemos usar a lei de Hooke para $\sigma_{\theta \theta}$.

$$
\sigma_{\theta \theta}=E \epsilon_{\theta \theta}=\frac{R_{n}-r}{r} \omega E=\frac{\omega E R_{n}}{r}-E \omega
$$

Substituindo a equação (7) nas equações (3) e (4) nos encontramos:

$$
\begin{aligned}
& N=R_{n} E \omega \int \frac{d A}{r}-E \omega \int d A=R_{n} E \omega A_{m}-E \omega A \\
& \begin{array}{r}
M_{x}=R_{n} R E \omega \int \frac{d A}{r}-\left(R+R_{n}\right) E \omega \int d A+E \omega \int r d A \\
=R_{n} R E \omega A_{m}-\left(R+R_{n}\right) E \omega A+E \omega R A=R_{n} E \omega\left(R A_{m}-A\right)
\end{array}
\end{aligned}
$$

Onde:

$$
A_{m}=\int \frac{d A}{r}
$$

A equação (9) pode ser reescrita da seguinte forma:

$$
R_{n} E \omega=\frac{M_{x}}{R A_{m}-A}
$$

Substituindo a equação (11) na equação (8) encontramos a seguinte relação:

$$
E \omega=\frac{A_{m} M_{x}}{A\left(R A_{m}-A\right)}-\frac{N}{A}
$$


Substituindo as equações (11) e (12) na equação (7) encontramos uma correlação para $\sigma_{\theta \theta}$ :

$$
\sigma_{\theta \theta}=\frac{N}{A}+\frac{M_{x}\left(A-r A_{m}\right)}{A r\left(R A_{m}-A\right)}
$$

Entretanto sabemos que para uma relação de $R / h$ grande o lado direito da equação (13) tende a $-M_{x} y / I_{x}$. Para provar esta redução notaremos que $r=R+y$, daí podemos reescrever o termo $R A_{m}$ da equação (13) como:

$$
R A_{m}=\int\left(\frac{R}{R+y}+1-1\right) d A=A-\int \frac{y}{R+y} d A
$$

Assim, para $R / h \rightarrow \infty$ o denominador da equação (13) fica:

$$
\begin{aligned}
& A r\left(R A_{m}-A\right)=-A \int\left(\frac{R y}{R+y}+y-y\right) d A-A y \int \frac{y}{(R+y) d A} \\
& \operatorname{Ar}\left(R A_{m}-A\right)=A \int \frac{y^{2}}{R+y} d A-A \int y d A-A y \int \frac{y}{R+y} d A \\
& \operatorname{Ar}\left(R A_{m}-A\right)=\frac{A}{R} \int \frac{y^{2}}{1+\frac{y}{R}} d A-A \int y d A-A y \int \frac{y}{1+\frac{y}{R}} d A
\end{aligned}
$$

Já que $R / h \rightarrow \infty, y / R \rightarrow 0,1+y / R \rightarrow 1, \int\left[y^{\wedge} 2 /(1+y / R) d A\right] \rightarrow I_{x}$ e $\int\left[\frac{y}{1+\frac{y}{R}} d A\right] \rightarrow$ 0, então encontramos o seguinte:

$$
\operatorname{Ar}\left(R A_{m}-A\right)=\frac{A I_{x}}{R}
$$

Assim o lado direito da equação pode ser rescrito da seguinte forma: 


$$
\frac{M_{x} R}{A I_{x}}\left(A-R A_{m}-y A_{m}\right)=\frac{M_{x} R}{A I_{x}}\left(\int \frac{\frac{y}{R}}{1+\frac{y}{R}} d A-\frac{y}{R} \int \frac{d A}{1+\frac{y}{R}}\right)=-\frac{M_{x} y}{I_{x}}
$$

Sendo assim a equação (13) pode ser reescrita da seguinte forma simplificada:

$$
\sigma_{\theta \theta}=\frac{N}{A}-\frac{M_{x} y}{I_{x}}
$$

Agora precisamos encontrar uma relação entre o momento $\left(M_{x}\right)$ e a força exercida no anel $(P)$. Como tais elementos são estaticamente indeterminados as equações de equilíbrio não são suficientes para determinar as resultantes internas $\left(V, N, M_{x}\right)$ nas seções dos membros. As relações adicionais são obtidas utilizando o teorema de Castigliano com as condições de contorno apropriadas.

Devido às propriedades simétricas do $\mathrm{AC}$ podemos analisar apenas o quadrante entre as seções IJ e KL (Figura (8)) para descobrir as relações necessárias, além disso, sabemos que quando o anel se deforma a seção KL se mantém perpendicular IJ. Com isso em mente, sabemos que pelo teorema de Castigliano que rotação na face $\mathrm{KL}\left(\phi_{K L}\right)$ é:

$$
\phi_{K L}=\frac{\partial U}{\partial M_{0}}=0
$$

Onde $U$ é a energia da tensão total e é definido pela seguinte relação:

$$
U=\int \frac{k V^{2} R}{2 A G} d \theta+\int \frac{N^{2} R}{2 A E} d \theta+\int \frac{A_{m} M_{x}^{2}}{2 A\left(R A_{m}-A\right) E} d \theta-\int \frac{M_{x} N}{E A}
$$

As cargas $V, N$, e $M_{x}$, que estão em um ângulo $\theta$ coma face $\mathrm{KL}$, podem ser determinadas pelas relações seguintes:

$$
V=\frac{P}{2} \sin (\theta)
$$


$N=\frac{P}{2} \cos (\theta)$

$M_{x}=M_{0}-\frac{P R}{2}(1-\cos \theta)$

Substituindo as equações (25) e (22) na equação (21):

$0=\int_{0}^{\pi / 2} \frac{\left[M_{0}-\left(\frac{P R}{2}\right)(1-\cos \theta)\right] A_{m}}{A\left(R A_{m}-A\right) E} d \theta-\int_{0}^{\pi / 2} \frac{\left(\frac{P}{2}\right) \cos \theta}{A E} d \theta$

Resolvendo a equação para $M_{0}$ :

$M_{0}=P R / 2\left(1-\frac{2 A}{R A_{m} \pi}\right)$

Entretanto, se $R / h>2$ nos podemos aproximar $U$ para uma de uma viga reta, assim nos substituímos o $3^{\circ}$ e o $4^{\circ}$ termo da equação (22) por:

$U_{m}=\int \frac{M_{x}^{2}}{2 E I_{x}} R d \theta$

Neste caso a relação que rege $M_{0}$ é:

$M_{0}=\frac{P R}{2}\left(1-\frac{2}{\pi}\right)$

Devido à natureza do problema, queremos que nossos sensores tenham o maior sinal possível como saída, e a partir das equações (29) e (25) percebemos que o maior momento aparece quando $\theta$ é nulo. Portanto podemos utilizar a equação (29) e a lei de Hooke na equação (20) para encontrar uma relação para $\epsilon$ máxima. 


$$
\epsilon=\frac{N}{E A}-\frac{y}{E I_{x}} \frac{P R}{2}\left(1-\frac{2}{\pi}\right)
$$

No nosso caso, a seção transversal possui uma área retangular constante ao longo do corpo e o momento de inércia dela é conhecido, $I_{x}=b h^{3} / 12$, assim como sua área, $A=b h$. Substituindo esses fatores e a equação (24) para $\theta=0$ na equação (30) encontramos:

$$
\epsilon=\frac{P}{2 b h E}-\frac{6 y P R}{b h^{3} E}\left(1-\frac{2}{\pi}\right)
$$

Assim temos uma relação direta entre a deformação e a força aplicada. 


\section{Calibração}

\subsection{Sensoriamento}

Para o sensoriamento do AC foram utilizados strain gages da Micro-Measurements (MM) do modelo EA-06-125MG-120. Dois pares do instrumento forma colados em cada face do anel, ou seja, um par na face interna e outro na face externa do mesmo lado. Está configuração foi escolhida para que possamos criar uma ponte de Wheatstone que amplifique o sinal de saída.

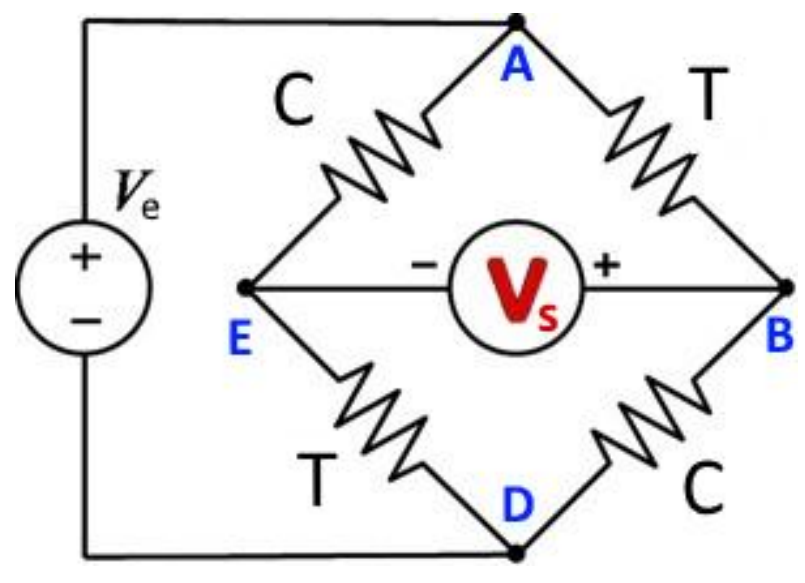

Figura (9): Ponte de Wheatstone

Para a colocação de tal sensor é preciso um processo laborioso de preparação da superfície. Primeiro a área onde foi escolhida para colocar o sensor tem que ser limpa com álcool e algodão, em seguida com uma lixa fina, de 220 grãos por centímetro quadrado, polimos a superfície do anel e depois limpamos novamente com acetona. Os strain gages são colados locais desejados, com Loctite 496, de forma que eles fiquem bem alinhados.

Além da colagem dos strain gages foi necessário colar e conectar um terminal para segurar a tensão da ponte. Assim, fios de cobre foram soldados as pontas de cada instrumento e conectados ao terminal. 


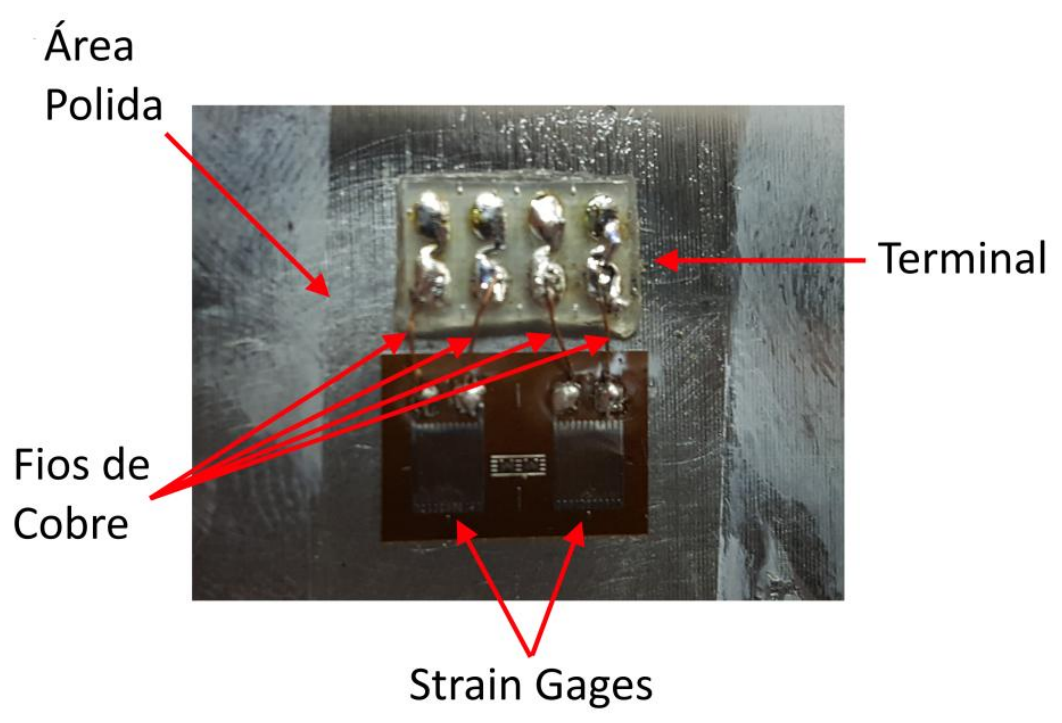

Figura (10): Strain gages e terminal colados no lugar.

A partir do terminal nos conectamos fios encapados para formar a ponte de Wheatstone mostrada na Figura (9), onde os strain gages externos, indicados com um "T" pois eles sofrem tração, estão cruzados com os internos, marcados com um “C”, que sofrem compressão.

A ponte de Wheatstone foi completa conectando mais dois terminais nas saídas dos strain gages de tração para que fosse possível levar um cabo coaxial de quatro fios para o instrumento de leitura, um P3 strain indicator and recorder da Visha Micro-Measurements.

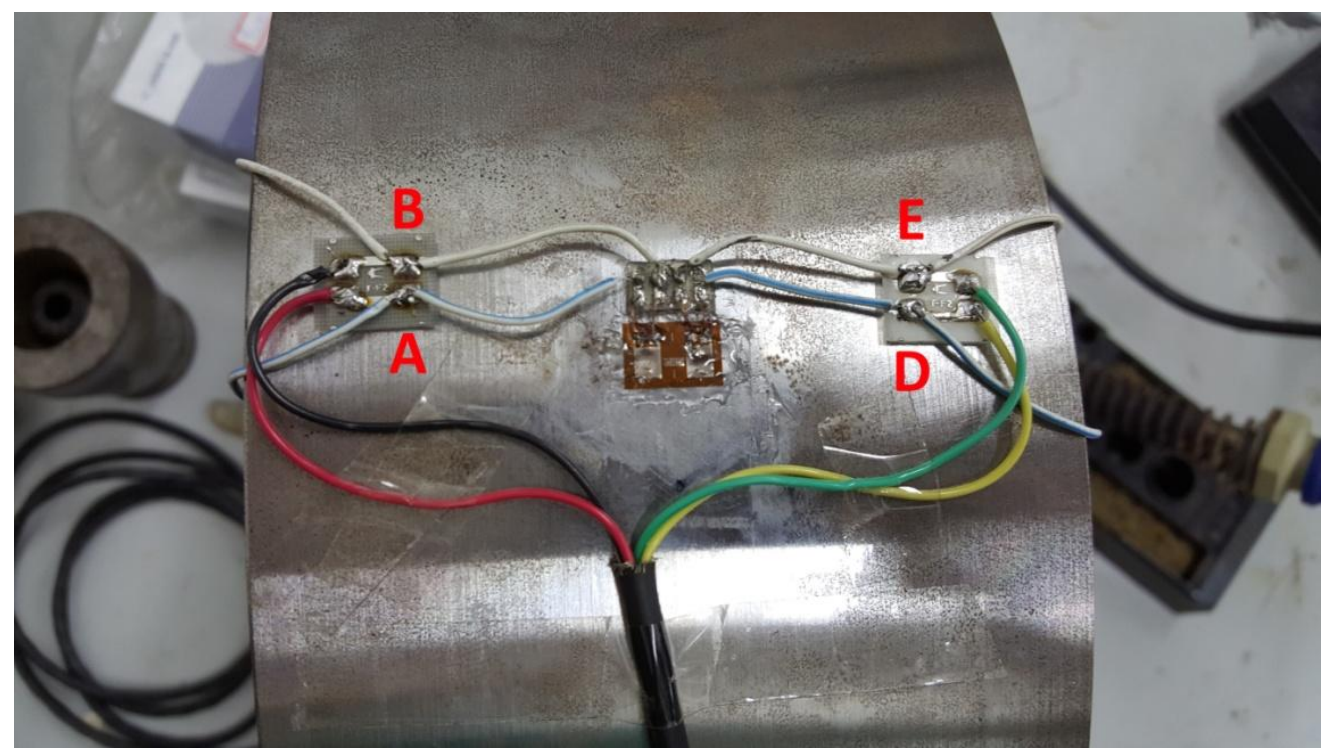

Figura (11): Strain gage conectado ao cabo coaxial. 
O cabo coaxial foi conectado no P3 como indicado nas instruções, de forma que os fios conectados as pontas A e D fossem conectados na alimentação, indicado por um $\mathrm{P}(+/-)$, de Power, e outras duas pontas, B e E, fossem conectadas a fonte de sinal, indicado por um $\mathrm{S}(+/-)$, de Signal, assim como mostra a Figura (12).

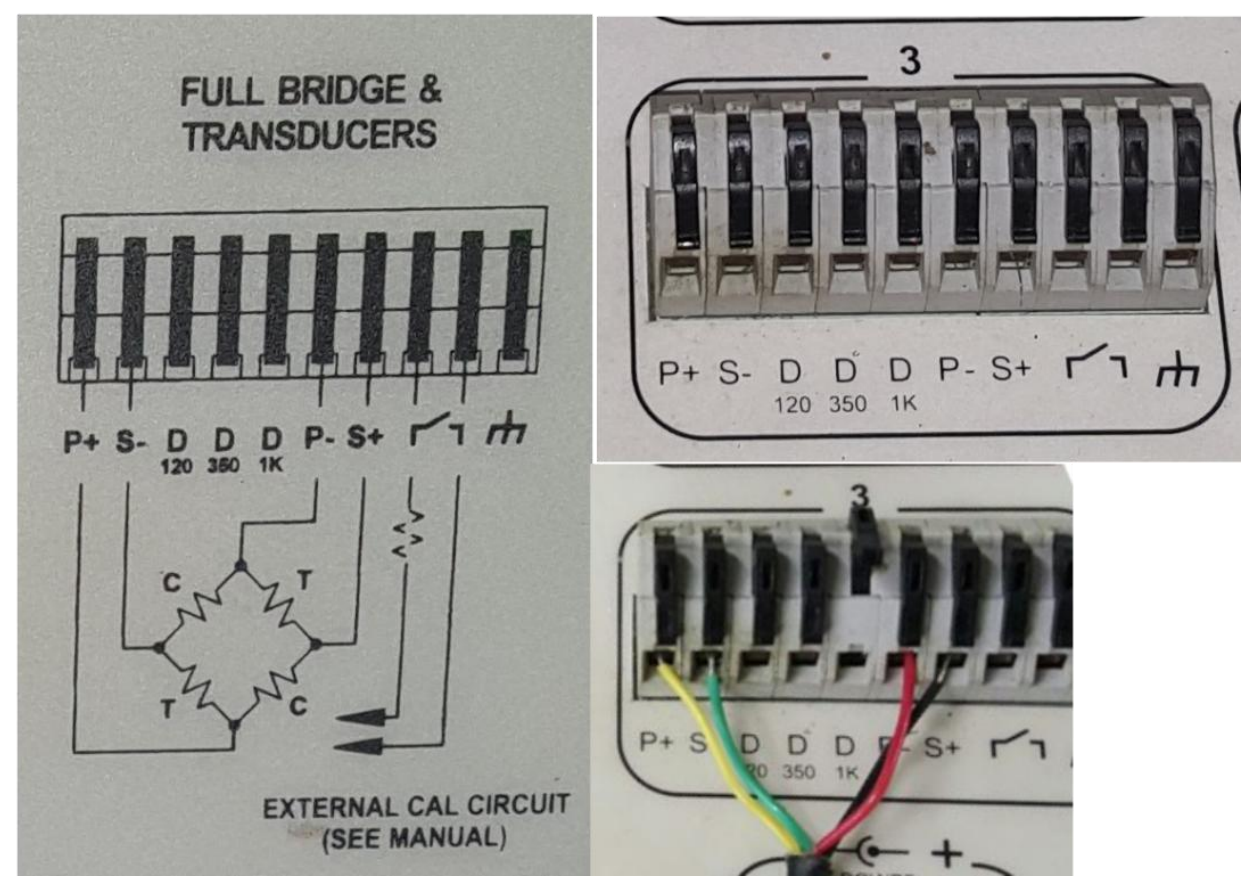

Figura (12): Conexões.

Para medirmos a deformação sofrida pelo AC a partir do sinal do instrumento é preciso converter a voltagem de saída $\left(V_{S}\right)$ para deformação $(\epsilon)$. Esta conversão é feita através de uma formula que utiliza a constante do gage factor $(G F)$ fornecida pelo fabricante. Assim sabemos que:

$$
\frac{\Delta R}{R_{0}}=G F \cdot \epsilon
$$

Onde $\Delta R$ é a variação na resistência e $R_{0}$ é a resistência nominal do strain gage. Compondo a relação dos resistores com a razão da voltagem de saída com a voltagem de entrada temos: 


$$
\frac{V_{s}}{V_{e}}=\frac{R_{1}}{R_{1}+R_{2}}-\frac{R_{4}}{R_{3}+R_{4}}
$$

Neste caso sabemos que se as resistências tiverem valores iguais a ponte estará balanceada e a relação terá o valor de zero. Entretanto se as resistências variarem existirá uma voltagem de saída, assim temos:

$$
\frac{V_{s}}{V_{e}}=\frac{1}{4}\left(\frac{\Delta R_{1}}{R_{1}}-\frac{\Delta R_{2}}{R_{2}}+\frac{\Delta R_{3}}{R_{3}}-\frac{\Delta R_{4}}{R_{4}}\right)
$$

Aplicando a equação (32) na equação (34):

$$
\frac{V_{s}}{V_{e}}=\frac{G F}{4}\left(\epsilon_{1}-\epsilon_{2}+\epsilon_{3}-\epsilon_{4}\right)
$$

Outro aspecto que também devemos esperar é que o modulo da deformação dos strain gages que estão sobre compressão, $\epsilon_{1}$ e $\epsilon_{3}$, seja o mesmo e além do mais seja negativo. Os que estiverem sobre tração também devem apresentar o mesmo modulo de deformação. Levando isso em consideração encontramos a seguinte equação:

$$
\frac{V_{s}}{V_{e}}=\frac{G F}{4}\left(2 \epsilon_{T}+2 \epsilon_{C}\right)
$$

Agora, com todos os parâmetros definidos, podemos calcular qual a força aplicada a partir da saída de voltagem do instrumento usando as equações (31) e (36). 


\subsection{Ensaio}

Para fazer a calibração manual do Anel de Carga usamos uma máquina de fuso, da Alfred J. Amsler \& Co disponível no laboratório do ITUC na PUC-Rio, que aplica tensão compressiva no anel. Assim escolhemos níveis discretos de tensão, que variam de $100 \mathrm{kgf}$ até $1000 \mathrm{kgf}$ em intervalos de $100 \mathrm{kgf}$. Este experimento foi repetido três vezes para garantir a equalização dos resultados. Para a nossa praticidade o equipamento $P 3$ estava já configurado para mostrar os resultados em Newtons e com isso coletamos os seguinte dados:

\begin{tabular}{r|rrrr}
\hline Força $(\mathbf{k g f})$ & T1(N) & T2(N) & T3(N) & T4(N) \\
\hline \hline $\mathbf{1 0 0}$ & 435 & 428 & 419 & 430 \\
$\mathbf{2 0 0}$ & 860 & 860 & 857 & 850 \\
$\mathbf{3 0 0}$ & 1300 & 1286 & 1281 & 1281 \\
$\mathbf{4 0 0}$ & 1730 & 1701 & 1700 & 1692 \\
$\mathbf{5 0 0}$ & 2148 & 2125 & 2125 & 2120 \\
$\mathbf{6 0 0}$ & 2572 & 2563 & 2550 & 2543 \\
$\mathbf{7 0 0}$ & 2977 & 2957 & 2957 & 2948 \\
$\mathbf{8 0 0}$ & 3400 & 3384 & 3397 & 3368 \\
$\mathbf{9 0 0}$ & 3838 & 3804 & 3804 & 3796 \\
$\mathbf{1 0 0 0}$ & 4263 & 4236 & 4235 & 4225 \\
\hline
\end{tabular}

Tabela (1): Resultados da calibração.

A primeira coisa que notamos é que existe um erro considerável entre a medida da máquina de fuso com o indicado no instrumento. Por exemplo, $100 \mathrm{kgf}$ deveria indicar uma leitura de 980,665 N, no entanto indica apenas $429 \mathrm{~N}$ de média. Entretanto se traçarmos um gráfico entre a média das medidas e a força aplicada percebemos que eles possuem uma relação linear, isso nos mostra que este erro pode ser corrigido por uma simples aritmética calibrando a saída do sinal. 


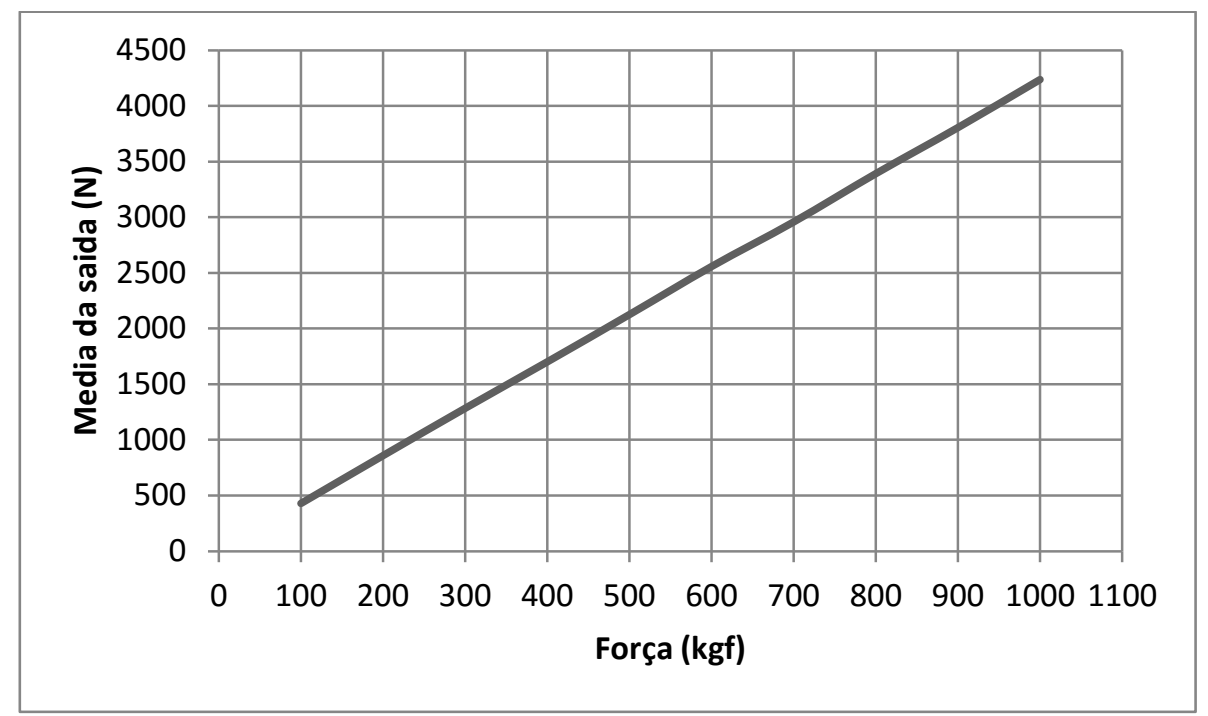

Figura (13): Gráfico da força aplicada com medida.

Calculando a média e o valor esperado, podemos inferir o desvio causado pela provável falta de calibração do equipamento, depois disso podemos ajustar as contas para calcular os erros relativos de indicação e o erro de reprodutibilidade.

\begin{tabular}{rrrrr}
\hline Média (N) & Força (N) & Fator de desvio & $\begin{array}{l}\text { Erro relativo de } \\
\text { indicação (\%) }\end{array}$ & $\begin{array}{l}\text { Erro } \\
\text { Reprodutivo de }\end{array}$ \\
\hline 429 & 980,665 & 2,285932401 & $-0,472435351$ & 3,734827264 \\
858,5 & 1961,33 & 2,284601048 & $-0,309542446$ & 1,169043722 \\
1283,5 & 2941,995 & 2,292165952 & $-0,518878452$ & 1,477679266 \\
1700,5 & 3922,66 & 2,306768597 & 0,147055069 & 2,23135643 \\
2125 & 4903,325 & 2,307447059 & 0,201186068 & 1,316036849 \\
2556,5 & 5883,99 & 2,301580286 & 0,158817702 & 1,135384856 \\
2957 & 6864,655 & 2,321493067 & 0,920829401 & 0,98059106 \\
3390,5 & 7845,32 & 2,313912402 & 0,816004482 & 0,945794172 \\
3804 & 8825,985 & 2,320185331 & 0,782245577 & 1,103057044 \\
4235,5 & 9806,65 & 2,315346476 & 0,636349829 & 0,896903323 \\
\hline
\end{tabular}

Tabela (2): Resultados.

Com isso podemos ver que basta multiplicar a força fornecida pelo $P 3$ por aproximadamente 2,3 que encontramos um erro aproximado bem pequeno. 


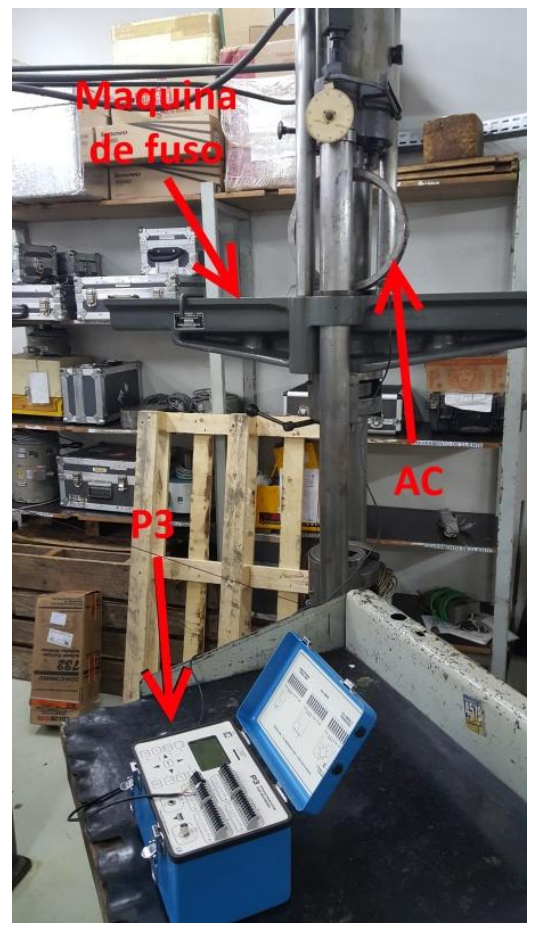

Figura (14): Teste na maquina de fuso. 


\section{Conclusão}

Sobre os resultados dos experimentos eu acredito que foram satisfatórios, pois os objetivos de construir e averiguar um AC foram alcançados, apesar de não ter sido possível fazer os testes de fadiga em meio corrosivo em um corpo de prova para qual o equipamento foi projeto.

A construção de um Anel de Carga apesar de ser aparentemente simples é um ótimo exercício de engenharia mecânica aplicada na pratica. Como pude ver na pela a experiência de projetar e construir um equipamento me levou em uma jornada de conhecimento onde tive que aplicar e reaprender diversos conhecimentos adquiridos no curso, dês das teorias de matérias até as técnicas de instrumentação. Além disso, também tive que aprender técnicas práticas, como a maneira de colar strain gages e como fazer soldas de fiação. No geral acredito que foi uma experiência enriquecedora e indispensável para a minha formação como engenheiro 


\section{$7 \quad$ Referencias Bibliográficas}

- Azom.com,. AISI 4140 Alloy Steel (UNS G41400). Disponível em: <http://www.azom.com/article.aspx?ArticleID=6769>. Acesso em: 26 fev. 2016.

- $\quad$ Boresi, A.; Sidebottom, O. Advanced mechanics of materials. Traducao . New York: Wiley, 1985.

- $\quad$ Cortest.com,. Proof Rings, Pyrex Containment Vessels, Elapsed Time Monitors, High Temperature Vessels, Tempressure Vessels. Disponível em: <http://www.cortest.com/proofring.htm>. Acesso em: 26 fev. 2016.

- de Castro, J. et al. Prediction of notch sensitivity effects in fatigue and in environmentally assisted cracking. Fatigue \& Fracture of Engineering Materials \& Structures, v. 38, n. 2, p. 161-179, 2014.

- $\quad$ de Castro, J.; Landim, R.; Meggiolaro, M. Defect tolerance under environmentally assisted cracking conditions. Corrosion Reviews, v. 33, n. 6, 2015.

- $\quad$ hoffmann, k. Applying the Wheatstone Brige Circuit. Traducao . 1. ed. [s.l: s.n.].

- $\quad$ Munnings, C.; Badwal, S.; Fini, D. Spontaneous stress-induced oxidation of Ce ions in Gd-doped ceria at room temperature. Ionics, v. 20, n. 8, p. 1117-1126, 2014.

- Wikipedia,. Strain gauge. Disponível em:

<https://en.wikipedia.org/wiki/Strain_gauge>. Acesso em: 26 fev. 2016.

- Wikipedia,. Stress intensity factor. Disponível em:

<https://en.wikipedia.org/wiki/Stress_intensity_factor>. Acesso em: 26 fev. 2016. 